\title{
Bilateral Serous Retinal Detachment as a Presenting Sign of Acute Lymphoblastic Leukemia
}

\author{
Jinseon Kim, Woohyok Chang, Min Sagong \\ Department of Ophthalmology, Yeungnam University College of Medicine, Daegu, Korea
}

\begin{abstract}
We present a case of bilateral serous retinal detachment (SRD) as a presenting sign of Philadelphia chromosome-positive acute lymphoblastic leukemia ( $\left.\mathrm{Ph}^{+} \mathrm{ALL}\right)$. A 45-year-old woman presented with decreased vision and was found to have bilateral serous retinal detachment. Peripheral blood smears revealed leukocytosis of $53.9 \times 10^{3} / \mu \mathrm{L}$ with $64.6 \%$ lymphoblasts. A bone marrow aspirate revealed the presence of lymphoblasts. Cytogenetic and molecular genetic analysis detected a reciprocal translocation between chromosome 9 and 22, $t(9 ; 22)$ (q34;q11). A diagnosis of $\mathrm{Ph}^{+} \mathrm{ALL}$ was made. Following systemic chemotherapy, the bilateral SRD resolved completely with full recovery of vision. The sudden appearance of SRD should raise suspicion for leukemia. Prompt recognition of this disease is important for early systemic treatment and restoration of visual function.
\end{abstract}

Key Words: Acute lymphoblastic leukemia, Precursor cell lymphoblastic leukemia-lymphoma, Serous retinal detachment

Ocular involvement is common in acute leukemia; it has been reported to occur in up to $90 \%$ of patients with this disease [1]. Typical ocular manifestations of leukemia are round or flame-shaped hemorrhages with a white component, intraretinal hemorrhages, and cotton wool spots, comprising what is called leukemic retinopathy. Serous retinal detachment (SRD) is a less common ocular manifestation of leukemia [1-3].

Several studies have reported serous macular detachment in lymphoproliferative disorders, but not as an initial symptom. In the majority of reported cases, the systemic disease had been diagnosed prior to the development of ocular symptoms [4-6]. Furthermore, reports of patients with acute lymphoblastic leukemia presenting with visual symptoms are rare.

We report a patient with Philadelphia chromosome-positive acute lymphoblastic leukemia $\left(\mathrm{Ph}^{+}\right.$ALL) who presented with bilateral SRD as the initial sign of leukemia. This is the first such reported case in Korea.

Received: January 19, 2009 Accepted: June 2, 2009

Reprint requests to Min Sagong. Department of Ophthalmology, Yeungnam University College of Medicine, \#317-1 Daemyeong-dong, Nam-gu, Daegu 705-717, Korea. Tel: 82-53-620-3440, Fax: 82-53-626-5936, E-mail: eyesgm@hotmail.com

* This study was presented as a poster at the 100th annual meeting of the Korean Ophthalmological Society in November 2008.

\section{Case Report}

A 45-year-old woman presented with a 10-day history of visual disturbance and bilateral central scotoma. This had been preceded by a one-month history of headache. Her corrected visual acuity was 0.2 in the right eye and 0.8 in the left eye. Slit lamp examination revealed no obvious inflammation in the anterior chamber or vitreous. Fundus examination of both eyes showed bilateral SRD in the posterior pole (Fig. 1A). Fluorescein angiography of both eyes showed multifocal hyperfluorescence beneath the detachment in the early phase and diffuse subretinal accumulation of fluorescein in the late phase (Fig. 1B). Stratus optical coherence tomography (OCT) showed serous retinal detachment with increased reflectivity in the choroid layers of both eyes (Fig. 1C). Neither neurologic nor dermatologic signs were present on physical examination. A blood test revealed leukocytosis of $53.9 \times 10^{3} / \mu \mathrm{L}$ with $64.6 \%$ lymphoblasts. The hemoglobin level was $8.5 \mathrm{gm} / \mathrm{dL}$ with a hematocrit of $24.9 \%$ and a platelet count of $208,000 / \mu \mathrm{L}$. The clotting screen was within normal limits, and the autoantibody screen was negative. Serum urate $(7.69 \mathrm{mg} / \mathrm{dL})$ and C-reactive protein $(0.421 \mathrm{mg} / \mathrm{dL})$ were within normal limits. Renal and liver function was normal.

Based on the ocular findings and hematological abnormalities, the patient was referred to a hematologist, who performed a bone marrow aspiration. Analysis of the aspirate showed 94\% lymphoblasts, confirming the diagnosis of ALL. Cytochemistry and immunophenotyping characterized the 

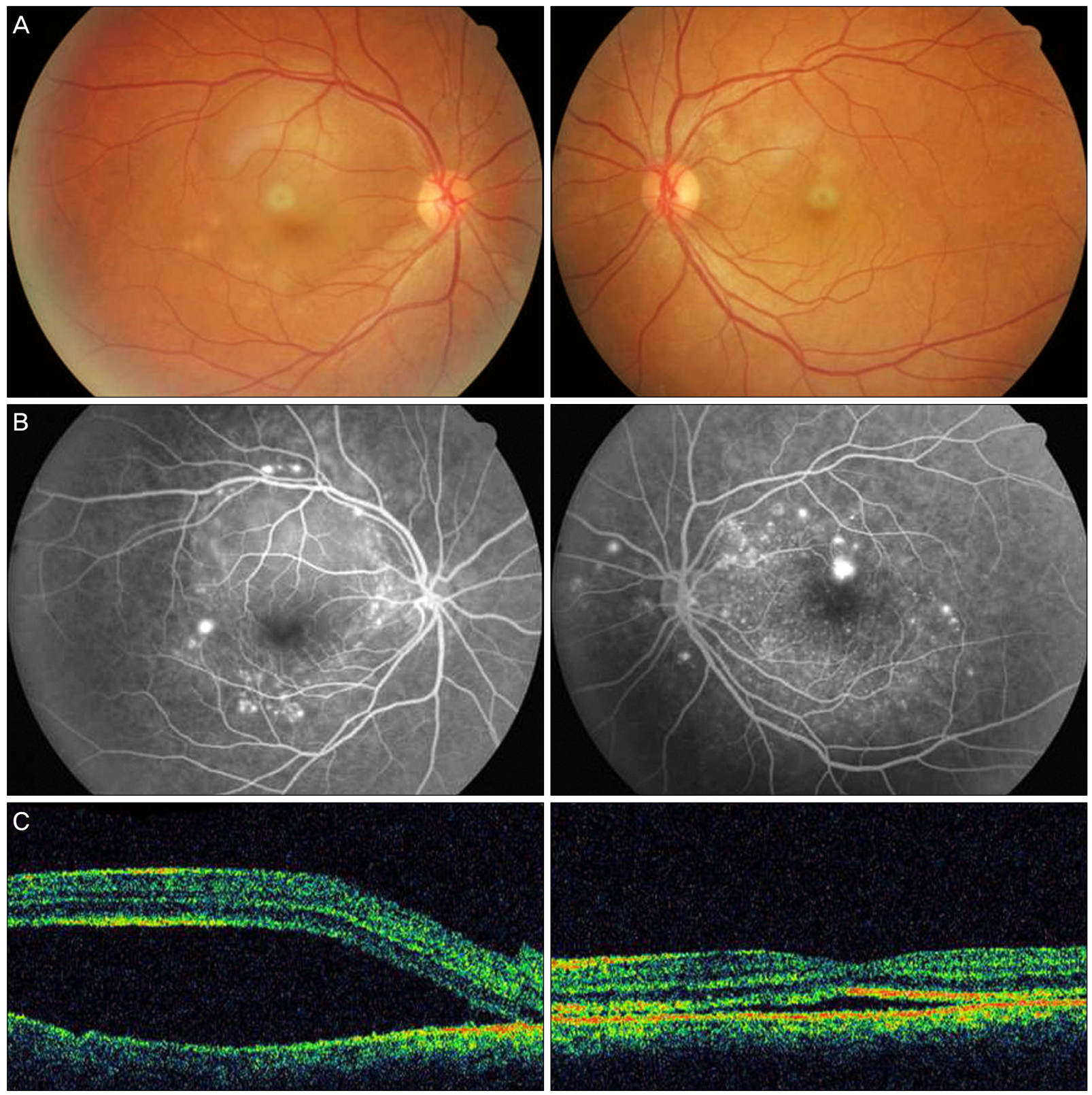

Fig. 1. (A) Fundus photographs of the right eye (left) and left eye (right) showing bilateral serous retinal detachment in the macula at the initial visit. (B) Corresponding fluorescein angiograms show diffuse subretinal accumulation of fluorescein with multifocal hyperfluorescent dots. (C) Corresponding optical coherence tomography scans show serous macular detachment.

leukemia as precursor B-cell ALL(L1). Cytogenetic and molecular genetic analysis showed $\mathrm{t}(9 ; 22)$ and minor bcr-abl fusion mRNA. The final diagnosis was $\mathrm{Ph}^{+}$ALL. Neurological examination, including lumbar puncture and magnetic resonance imaging, showed no involvement of the central nervous system. The patient was enrolled in a course of remission induction chemotherapy consisting of intravenous daunorubicin, vincristine, and methylprednisolone. One month after induction therapy was initiated, she was treated with consolidation chemotherapy consisting of intravenous methotrexate, cytarabine and methylprednisolone. Intrathecal methotrexate was administrated as prophylaxis for central nervous system involvement. Thereafter, two additional cycles of consolidation chemotherapy were administered. Throughout the whole treatment period, the patient was also given imatinib once daily to inhibit the activity of the bcr-abl fusion protein.

One month after induction therapy was initiated, fundus examination, fluorescein angiography, and OCT showed complete resolution of the bilateral SRD (Fig. 2). Visual acuity had improved to 1.0 in both eyes. Four months after the initiation of induction therapy, the patient was in complete remission with no ocular symptoms. 

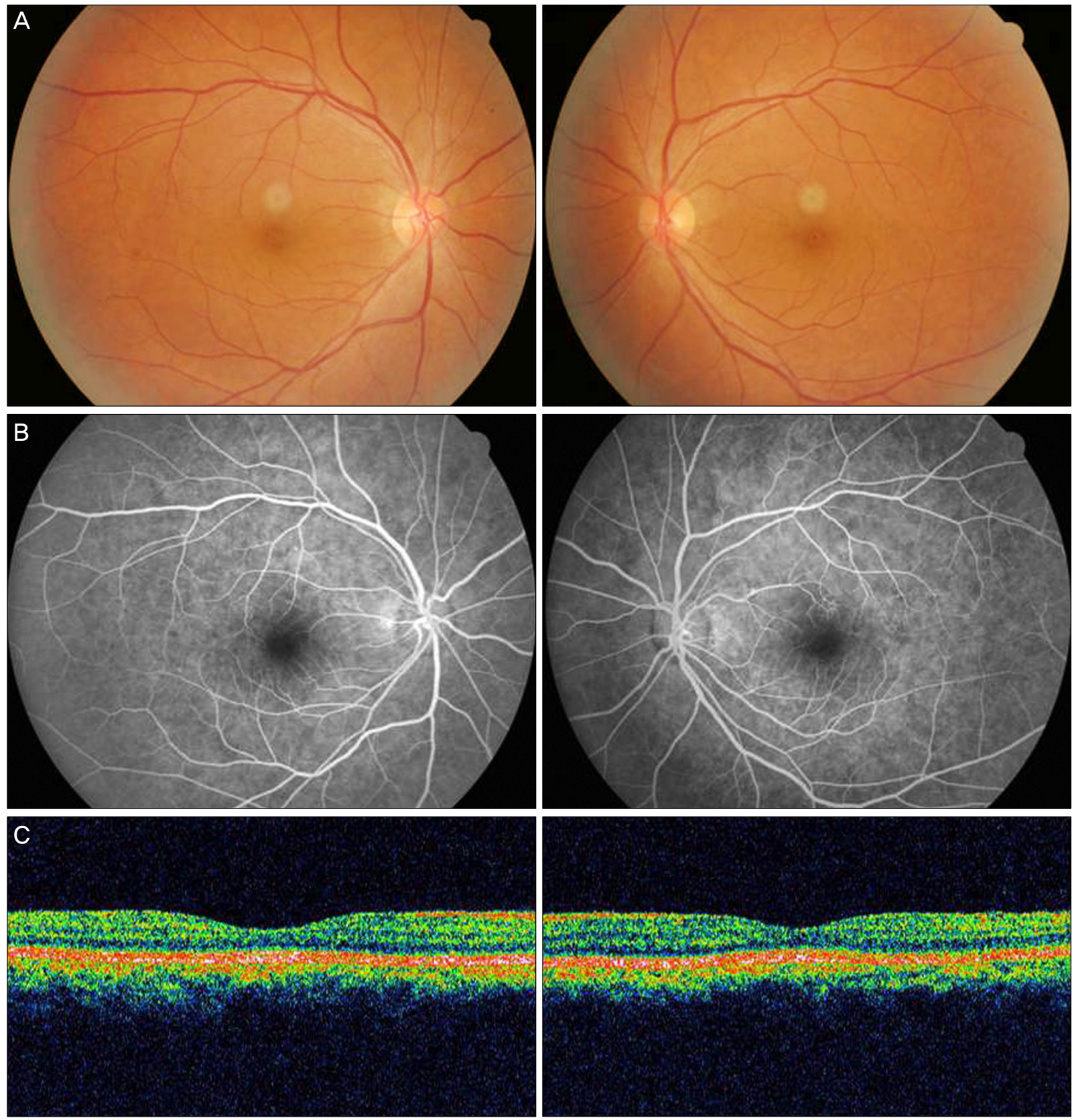

Fig. 2. (A) Fundus photographs of the right eye (left) and left eye (right) showing complete resolution of bilateral serous retinal detachment one month after systemic chemotherapy. (B) Corresponding fluorescein angiograms show no subretinal accumulation of fluorescein. (C) Corresponding optical coherence tomography scans show nearly normal foveal anatomy.

\section{Discussion}

Ocular changes in leukemia are varied and may involve all ocular and adnexal tissues [7]. Most ocular manifestations occur secondary to direct leukemic infiltration of the ocular structures; hematologic abnormalities such as thrombocytopenia, anemia, leucopenia, or hyperviscosity; opportunistic infections; or secondary to the treatment of the leukemia itself $[7,8]$. In the fundus, common manifestations include retinal vascular sheathing, cotton wool spots, white-centered retinal hemorrhages, nodular infiltrates, and optic nerve head infiltrates $[1,7,9]$. These fundus changes are common during the course of the disease; they have been reported to occur in up to $90 \%$ of patients with leukemia [1]. However, SRD has been reported less commonly in the setting of acute leukemia [1-3]. SRD is even less common as the initial finding in this disease. In the majority of cases, systemic disease has already been diagnosed prior to the development of ocular symptoms [4-6]. Most of the previously reported cases of ALL with SRD have involved younger patients $[6,10,11]$. Our patient was a 45 -year-old woman whose visual impairment was caused by bilateral SRD and led to a diagnosis of 
ALL.

SRD in the setting of leukemia has been reported to be shallow in the posterior pole $[3,10]$. Fluorescein angiographic findings in previous studies have demonstrated multifocal hyperfluorescence beneath the detachment in the early phase and diffuse subretinal accumulation of fluorescein in the late phase $[3,10]$; we noted similar findings in our case. SRD may develop as a result of choroidal involvement by leukemic cells causing retinal pigment epithelial disturbances or due to incompetence of the outer blood-retinal barrier inducing retinal pigment epithelial changes $[3,12]$.

The differential diagnosis of SRD includes Harada's syndrome, central serous choroidopathy, uveal effusion syndrome, age-related macular degeneration, choroidal hemangioma, and metastatic neoplasm. Bilateral SRD and multifocal hyperfluorescence beneath the detachment in the early phase of fluorescein angiography would support Harada's syndrome as the most likely diagnosis. However, our case showed no inflammatory reaction in the anterior chamber or vitreous. Furthermore, the absence of neurologic and dermatologic signs, such as dysacusis, vitiligo, and poliosis, is not consistent with Harada's syndrome.

Intraocular manifestations of leukemia are not usually treated directly. Systemic chemotherapy is recommended first. When definite leukemic infiltrates fail to respond promptly to chemotherapy, ocular radiation is implemented [1]. In our case, the patient received only systemic chemotherapy, and the SRD resolved completely with full recovery of visual acuity. This suggests that leukemic cells had infiltrated into the choroid in the area of the SRD. Therefore, we assume that the SRD was a manifestation of ALL. This also suggests that systemic chemotherapy alone can preserve visual acuity if performed early.

Our patient had $\mathrm{Ph}^{+}$ALL. Recent advances in therapy have resulted in better survival for ALL patients. However, older age and specific cytogenetic abnormalities, such as the Philadelphia chromosome, a reciprocal translocation between chromosome 9 and 22, $\mathrm{t}(9 ; 22)(\mathrm{q} 34 ; \mathrm{q} 11)$, are adverse prognostic factors in ALL [13]. Therefore, early diagnosis and appropriate treatment are essential for securing a favorable prognosis, especially in high-risk ALL patients. Our case illustrates that prompt treatment after diagnosis, even in a patient with $\mathrm{Ph}^{+} \mathrm{ALL}$, can result in complete reso- lution of SRD and allow for full restoration of visual function.

In conclusion, bilateral SRD may be the first sign of ALL. Thus, ophthalmologists should consider a thorough systemic evaluation for leukemia in patients with sudden SRD.

\section{Conflict of Interest}

No potential conflict of interest relevant to this article was reported.

\section{References}

1. Kincaid MC, Green WR. Ocular and orbital involvement in leukemia. Surv Ophthalmol 1983;27:211-32.

2. Tang RA, Vila-Coro AA, Wall S, et al. Acute leukemia presenting as a retinal pigment epithelium detachment. Arch Ophthalmol 1988;106:21-2.

3. Stewart MW, Gitter KA, Cohen G. Acute leukemia presenting as a unilateral exudative retinal detachment. Retina 1989;9: $110-4$.

4. Burns CA, Blodi FC, Williamson BK. Acute lymphocytic leukemia and central serous retinopathy. Trans Am Acad Ophthalmol Otolaryngol 1965;69:307-9.

5. Murphy JA, Pitts JF, Dudgeon J, et al. Retinal detachments due to chronic lymphocytic leukaemia. Clin Lab Haematol 1991;13: 217-20.

6. Reddy SC, Menon BS. A prospective study of ocular manifestations in childhood acute leukaemia. Acta Ophthalmol Scand 1998;76: 700-3.

7. Rosenthal AR. Ocular manifestations of leukemia: a review. Ophthalmology 1983;90:899-905.

8. Wu L, Calderon M, Hernandez G, et al. Bilateral exudative retinal detachment as the first sign of relapsing acute myelogenous leukaemia. Clin Experiment Ophthalmol 2006;34:623-5.

9. Park YK, Jun RM, Ahn CS. A case of optic nerve infiltration of acute lymphocytic leukemia. J Korean Ophthalmol Soc 2000; 41:2008-12.

10. Miyamoto K, Kashii S, Honda Y. Serous retinal detachment caused by leukemic choroidal infiltration during complete remission. Br J Ophthalmol 2000;84:1318-9.

11. Malik R, Shah A, Greaney MJ, et al. Bilateral serous macular detachment as a presenting feature of acute lymphoblastic leukemia. Eur J Ophthalmol 2005;15:284-6.

12. Hine JE, Kingham JD. Myelogenous leukemia and bilateral exudative retinal detachment. Ann Ophthalmol 1979;11:1867-72.

13. Faderl S, Jeha S, Kantarjian HM. The biology and therapy of adult acute lymphoblastic leukemia. Cancer 2003;98:1337-54. 